Mongelli, B. P. - Zur Inversion des Subjektbegriffs

\title{
Zur Inversion des Subjektbegriffs im medizinisch- literarischen Kontext Georg Büchners
}

\author{
Boris Previsic Mongelli'
}

\begin{abstract}
The purpose of this paper is to compare Büchner's medical and literary discourse, namely Probevorlesung über Schädelnerven and Woyzeck. Both texts were written towards the end of the author's short life. The comparative analysis provides evidence that Büchner inverts the notions of subjectivity and objectivity; he lets them 'go on their head', "auf dem Kopf gehn" (Lenz). In his medical lecture, Büchner demonstrates free will, whereas in the theatre piece, especially in the doctor's scene, he shows the subjection of Woyzeck. As this paper will show, by taking into consideration the political awareness of revolutionary movements like the "Vormärz", it is possible to make a critical reflection about dependency and autonomy of the 'subject of history'.
\end{abstract}

Keywords: Büchner; Woyzeck; medicine; subject

Zusammenfassung: In diesem Aufsatz werden der medizinische und literarische Diskurs bei Büchner miteinander verglichen. Vor allem in seiner Zürcher Probevorlesung über Schädelnerven und in seinem Woyzeck, welche im letzten Lebensjahr Büchners entstehen, scheinen die Begriffe von Subjektivität und Objektivität - in Lenzscher Anlehnung - ,auf dem Kopf [zu] gehn“. In seiner medizinischen Vorlesung beweist der Privatdozent Büchner, dass es den freien Willen gibt, während er in seinem Woyzeck, insbesondere in der Doktorszene, die Unterdrückung des Protagonisten eindrücklich in Szene setzt. Dieser Aufsatz zeigt somit auf, dass eine kritische Reflexion über die Abhängigkeit und den freien Willen des „Subjekts der Geschichte“ auf der Folie der politischen Sensibilisierung in Umbruchzeiten wie im Vormärz möglich wird.

Stichwörter: Büchner; Woyzeck; Medizin; Subjekt

Die Inversion des Subjekt- und Objektbegriffs im Woyzeck hat etwas mit Büchners wissenschaftlichem Schaffen zu tun; doch das eine als Reaktion auf das andere oder das andere als Reaktion auf das eine zu deuten, scheint kein einfaches Unterfangen zu sein. Bevor

\footnotetext{
${ }^{1}$ Doktor der Neueren Deutschen und Allgemeinen Literaturwissenschaft am Lehrstuhl Alexander Honold am Deutschen Seminar der Universität Basel. Email: boris.previsic@unibas.ch
} 
Mongelli, B. P. - Zur Inversion des Subjektbegriffs

das Betätigungsfeld Büchners ganz nach dem Titel der Büchner-Monographie von VIËTOR (Georg Büchner. Politik. Literatur. Wissenschaft, 1949) überhaupt umrissen werden kann und Verbindungen zwischen den einzelnen Disziplinen hergestellt werden, muss auf den letzten Punkt, auf die wohl arbeitsintensivste Disziplin der Trias, eingegangen werden - auf die Wissenschaft. Dank der materialreichen, 2008 erschienenen Marburger Ausgabe von Büchners Naturwissenschaftliche(n) Schriften (NS) ist dieses Arbeitsgebiet des Autors nun gut zugänglich geworden.

Obwohl Georg Büchner zunächst den Fußstapfen seines Vaters zu folgen scheint und sich für ein Medizinstudium in Strassburg 1831 einschreibt, so interessiert ihn immer weniger die „Arzeneykunst“ als vielmehr die allgemeineren Fächer Naturwissenschaft und Philosophie (welche heute in abgewandelter Form innerhalb des Studium generale ein Mauerblümchendasein fristet). Wenn er sich fünf Jahre später in Zürich um eine Privatdozentenstelle bewirbt, so will er in erster Linie Vorlesungen über Philosophie halten; er habe - wie er im Brief vom 31. Mai oder 1. Juni an Gutzkow formuliert - „die fixe Idee, im nächsten Semester zu Zürich einen Kurs über die Entwickelung der deutschen Philosophie seit Cartesius zu lesen“ (NS: 213). Das Sprungbrett dazu bietet ihm seine Mémoire, mit welcher er seine Fähigkeiten in vergleichender Anatomie und Zoologie unter Beweis stellt und auf deren Grundlage er auch seine Probevorlesung bestreitet. Als vergleichender Anatome bewegt sich Büchner im wohl wichtigsten Fach seiner Zeit. Man könnte sagen, er sei das, was der Genforscher bzw. der Neurowissenschaftler heute ist, im ersten Drittel des 19. Jahrhunderts. Gemäss dem philosophisch-medicinischen Wörterbuch (1807) besteht das Ziel dieser Wissenschaft darin, anhand vergleichender anatomischer und physiologischer Studien die ,animalischen Erscheinungen als eine Natur d.i. als ein gesetzmäßig verknüpftes Ganzes von Gegenständen unserer Wahrnehmung“ (NS: 190) philosophisch zu erfassen. Damit wird die Trennung zwischen der empirischen Naturwissenschaft und den theoretisch angelegten „medicinischen-philosophischen“ Wissenschaften unscharf. Gerade in dieser Unschärferelation zwischen harter und weicher Wissenschaft liegt das Interesse Büchners, wenn er seine ersten Skripte zur vergleichenden Anatomie von Cartesius einerseits und zur Philosophie Spinozas andererseits verfasst. ${ }^{2}$

\footnotetext{
${ }^{2}$ Wie sein Bruder Ludwig Büchner berichtet, ,,präparirte [Georg], um mit zwei Fächern ausgerüstet nach Zürich zu kommen, einen vollständigen Lehrkurs über, die philosophischen Systeme der Deutschen seit Cartesius und Spinoza"“ (NS: 213).
} 
Mongelli, B. P. - Zur Inversion des Subjektbegriffs

Am 5. November 1836 hält Büchner seine Probevorlesung vor „circa 20 Zuhörern“ (NS: 217). Zum grössten Teil übernimmt er einfach Teile aus seiner Mémoire, welche er lediglich übersetzt. Sie wird nur durch die Einleitung und die Bezugnahme auf Friedrich Arnolds Referat Der Kopftheil des vegetativen Nervensystems erweitert, welches er ausführt (NS: 157-159). Darin heisst es unter anderem: „Auf gleiche Weise scheint helles Licht Niesen hervorzubringen, indem sich die Reizung der Blendungsnerven $\mathrm{zu}$ den mit ihnen verbundenen Nasennerven oder die des Sehnerven durch die Verbindung mit dem Nasenknoten auf dem obigen Wege zu dem nervus phrenicus fortpflanzt.“ (nach NS: 217) Diese Stelle hinterlässt Spuren in der ebenfalls in Zürich geschriebenen Szene im Woyzeck, in welcher der „Doctor“ seiner titelgebenden Versuchsperson vorwirft, einfach so „auf die Wand“ gepisst zu haben: „Ich hab's gesehn, mit diesen Augen gesehn, ich streckte grade die Nase zum Fenster hinaus u. ließ die Sonnestrahlen hinein fallen, um das Niesen zu beobachten." (Büchner 1988: H4,8, 226) ${ }^{3}$ Mit anderen Worten: In dem Moment, in dem der Doktor einen Eigenversuch anstellt, um eine Theorie des Niesens zu bestätigen, welche auf einer synästhetischen Verbindung zwischen Sehnerv und Nasennerv basiert, entgeht ihm der zwar vertraglich gesicherte, wertvolle und später $\mathrm{zu}$ analysierende Urin des Menschenversuchs. ${ }^{4}$ So sehr Büchner offenbar die Figur des Doktors ironisierend behandelt, so eng ist die Probevorlesung zumindest mit der letzten Entwurffassung verknüpft. Auf solche Weise wird die intertextuelle Beziehung zwischen literarischem und naturwissenschaftlichem Schaffen im letzten Lebensjahr Büchners besiegelt.

Gerade der einleitende Teil der Probevorlesung ist nicht nur als Frucht seines SpinozaSkripts, sondern auch als Fortführung der gestrichenen naturwissenschaftlichen

\footnotetext{
3 Die Szenenbezeichnung bezieht sich auf die auch in die historisch-kritische Marburger Ausgabe übernommenen Siglen, welche die Nummer des Handschriftkonvoluts, die Entwurfsstufen (H1 bis H4) und die jeweilige Szene darin verzeichnet, die Seitenangaben hingegen auf die im folgenden auch zitierte Münchner Ausgabe (BÜCHNER 1988).

${ }^{4}$ In der ersten Entwurfsstufe ist dieser gegenseitige Bezug noch nicht offensichtlich, wenn der Doktor sagt: „Aber ich hab's gesehen, dass er an die Wand pißte, ich steckt grad mein Kopf hinaus, zwischen meiner Valnessia u.Myriaden“ (BÜCHNER 1988: H1,6, 213). Udo Roth zeigt schlüssig auf, wie sich hier der Doktor ernsthaft mit Grundlagenforschung im Sinne der genetischen Methode beschäftigt (ROTH 2000). Wenn man will, so ersetzt hier Büchner die kantische Apriorifunktion, auf deren Basis die Beobachtung der Grundlagenforschung stattzufinden hat, systemtheoretisch avant sa lettre durch eine Beobachtung zweiter Ordnung: „Die Beobachtung des Einen im Einen müsste aber das, was sie ausschließt [...], einschließen.“ Dies wiederum erfordert „einen imaginären Raum (so wie man von imaginären Zahlen spricht), und dieser imaginäre Raum tritt an die Stelle des klassischen Apriori der Transzendentalphilosophie.“ Daran kann sich die „Bedeutung des Beobachtens zweiter Ordnung“ anschliessen, und die Schlussfolgerung lautet: „Statt auf letzte Einheiten zu rekurrieren, beobachtet man Beobachtungen, beschreibt man Beschreibungen."(LUHMANN 1990: 716f.)
} 
Mongelli, B. P. - Zur Inversion des Subjektbegriffs

Grundlagenforschung in der Doktorszene zu betrachten. Bewusst positioniert er sich - im Unterschied $\mathrm{zu}$ seiner Mémoire - gegen einen teleologischen Funktionalismus, der ,in England und Frankreich“ überwiege (NS: 153). Dieser anglo-französische Funktionalismus kenne „das Individuum nur als etwas, das einen Zweck außer sich erreichen soll, und nur in der Bestrebung, sich der Außenwelt gegenüber theils als Individuum, theils als Art zu behaupten“ (NS: 153). Gegen dieses funktionalistische Modell stellt Büchner die im deutschen Sprachraum entwickelte genetische Methode, in welcher „Alles nach einer gewissen Einheit, nach dem Zurückführen aller Formen auf den einfachsten primitiven Typus“ strebe (NS: 155). Im Rückgriff auf „die Repräsentationsidee Oken's in der Klassification des Thierreichs“ (NS: 155) entwickelt er sich sein eigenes Modell. Damit bringt er die gestrichene Szene durch die Hintertüre einer Einleitung wieder in die Diskussion. In diesem Zusammenhang ist es auch festzustellen, dass eigentlich erst die Zusammenführung dieser zwei Standpunkte, des teleologischen einerseits, der unter anderem auch das Überleben des Lebenstüchtigeren anvisiert, und des genetischen andererseits, der auf einer Genealogie der Tierarten basiert, zum späteren Darwinschen Modell führen.

Damit schreibt sich Büchner in die aktuelle Debatte ein und umgeht von vornherein das Dilemma, welches er noch in seiner Dissertation im Übergang vom beschreibenden zum philosophischen Teil hatte, wo die unzähligen Tableaux der Interpretation bedürfen und nur qua nachträglicher nominalistischer Potenz die „genetische Methode“ wieder fruchtbar macht ${ }^{5}$; nicht dass er die „teleologische Methode“ in Bausch und Bogen verwürfe. Die kennt er zu gut aus seinem Studium, als dass er sich diese nicht zunutze machen würde. Aber er argumentiert, dass das Lebewesen nicht einfach als „,verwickelte Maschine“ betrachtet werden darf (NS: 153), denn:

Die Natur handelt nicht nach Zwecken, sie reibt sich nicht in einer unendlichen Reihe von Zwecken auf von denen der eine den anderen bedingt; sondern sie ist in allen ihren Aeußerungen sich unmittelbar selbst genug. Alles, was ist, ist um seiner selbst willen da. Das Gesetz dieses Seins zu

\footnotetext{
${ }^{5}$ „Die partie descriptive [...] kulminiert im hybriden Medium von Bildern, die gelesen werden wollen. [...] In dieser darstellerischen Sackgasse befindet sich nicht nur Büchner, sondern die beschreibende Anatomie allgemein." Der Übergang gelingt einem textlich-literarischen Verfahren der partie philosophique in Büchners Dissertation, in welcher „,die Hirnnerven in einem idealen Gesamtkörper [...], der zeitlicher Natur ist“ vorgeführt werden. Die Potenzierung des Experiments erfolgt qua Autorität sprachlicher Verweisung zeitlich nach dem Experiment. Entsprechend inflationär verwendet Büchner das Verb „répondre“ im ,philosophischen Teil’ seiner Dissertation (MÜLLER-SIEVERS 2003: 80ff.).
} 


\title{
Mongelli, B. P. - Zur Inversion des Subjektbegriffs
}

suchen, ist das Ziel der der teleologischen gegenüberstehenden Ansicht, die ich die philosophische nennen will. Alles, was für jene Zweck ist, wird für diese Wirkung. Wo die teleologische Schule mit ihrer Antwort fertig ist, fängt die Frage für die philosophische an. (NS: 153-155)

Zweierlei Sachverhalte sind aus dieser Stelle zu lesen: Erstens reiht sich Büchner damit sicherlich auch um seine Berufsaussichten zu sichern - pragmatisch geschickt in eine Traditionslinie ein, welche er in Zürich vorfindet; es handelt sich dabei vor allem um die Schädelwirbeltheorie, welche Oken propagiert, indem er die Metamorphose zwischen Hörorgan und Fischkiemen postuliert. Zweitens verknüpft er diese genetische Methode mit einer philosophischen, für die „die Frage“ erst anfängt. Zwar hält Büchner nicht viel von einer apriorischen Form von Spekulation in der philosophisch orientierten Naturwissenschaft, wie sie von Carus und zum Teil auch von Oken vertreten wird. Dies zeigt sich ebenfalls in der Einleitung der Probevorlesung, wo er sarkastisch anfügt, die „Philosophie a priori“ sitze „noch in einer trostlosen Wüste“; sie habe „einen weiten Weg zwischen sich und dem frischen grünen Leben“ und es sei „eine große Frage, ob sie ihn je zurücklegen“ werde (NS: 155). Dennoch sieht er eine Zweckfreiheit in der Natur zugunsten einer übergeordneten „Harmonie“. In seinen Worten:

\begin{abstract}
Alles, Form und Stoff, ist für [die reinsten Formen] an dies [Ur-]Gesetz gebunden. Alle Funktionen sind Wirkungen desselben; sie werden durch keine äußeren Zwecke bestimmt, und ihr sogenanntes zweckmäßiges Aufeinanderund Zusammenwirken ist nichts weiter, als die nothwendige Harmonie in den Aeußerungen eines und desselben Gesetzes, dessen Wirkungen sich natürlich nicht gegenseitig zerstören. (NS: 155)
\end{abstract}

Dieses „Urgesetz“ kann im Zusammenhang mit Spinozas Ethik gelesen werden, welche nach Euklids geometrischen Grundbegriffen geordnet ist und sich am pantheistisch übergeordneten Gesetz Gottes orientiert. Dieses unendliche Wesen als „natura naturans“ schöpft die „,natura naturata“ von selbst. Die dualistische Auffassung Descartes' wird so auf eine monistische konzentriert, in der Seele und Leib eine Einheit bilden. Alles unterliegt der Unabänderlichkeit diese „Urgesetzes“, selbst Gott. Innerhalb dieses Systems gibt es darum keine Zweckursachen 
Mongelli, B. P. - Zur Inversion des Subjektbegriffs

und demnach auch keine Willensfreiheit, da selbst der Mensch immer nur als der Welt immanentes Wesen aufgefasst werden kann. ${ }^{6}$

Gerade aus der Kritik an der naturwissenschaftlichen „Philosophie a priori“, welche sich den Gegenstand anhand ihrer Theorie zurechtzulegen versucht und dadurch neue Erkenntnisse aus der Naturwissenschaft - wie seinerzeit beispielsweise des Blutkreislaufs verkennt, kann sich Büchner vollumfänglich auf ein empirisches Vorgehen der Naturwissenschaft einlassen, da er seine Argumentation mit der neuen Einleitung bereits in die richtigen Bahnen gelenkt hat. Die Empirie setzt er darum nicht zugunsten eines Funktionalismus wie in Frankreich oder in England, sondern zugunsten des höheren Prinzips ein. So verschränkt er die Methode der einen Richtung (die empirische Methode der funktionalen Richtung) mit dem Ziel der anderen, der philosophischen Richtung; notabene sind das genau die beiden Richtungen, die er eingangs seiner Probevorlesung scharf trennt. Nur schon ein Blick auf den Quellencorpus seiner Mémoire zeigt, dass er sich überhaupt nicht auf eine Nationalrichtung einschießt, sondern - im Gegenteil - seine Thesen international mit Fachliteratur aus ganz Europa auf breiter Basis abstützt. So kann man mutmaßen, ob es an seiner Herkunft aus der deutschen Tradition einerseits, seiner wesentlichen Schulung in Frankreich unter einem Cuvier-Schüler, nämlich Duvernoy, andererseits liegt, dass es ihm möglich wurde, vielversprechende ,methodologische Ansätze aus Deutschland wie zum Beispiel die genetische Methode mit der sehr viel stärker empirisch und funktionalistisch ausgerichteten Tradition in Frankreich zu verbinden“ (NS: 253).

Verfolgt man nun seine Beweisführung in der Mémoire, woraus die Probevorlesung in der Folge nur noch übersetzend zitiert, so zeigt sich, mit welcher akribischen Empirie er vorgeht, um im Sinne der genetischen Methode den einheitlichen Bauplan der Barbe gerade

\footnotetext{
${ }^{6}$ Prägnant fällt Žižeks Folgerung von dieser Auffassung Spinozas, wie sie im Tractatus theologico-politicus aus dem Jahre 1670 formuliert wird, aus: „Für Spinoza gibt es kein Hobbessches ,Selbst', das der Wirklichkeit entzogen wäre und ihr gegenüberstünde. Spinozas Ontologie ist die Ontologie vollkommener Immanenz in der Welt - d.h. ich ,bin' nichts als das Netzwerk meiner Beziehungen zur Welt und in ihm vollkommen ,entäußert'. Mein conatus, mein Streben, mich selbst zu behaupten, ist somit keine Selbstbehauptung auf Kosten der Welt, sondern mein uneingeschränktes Akzeptieren der Tatsache, dass ich Teil der Welt bin, mein Zur-GeltungBringen der umfassenderen Wirklichkeit, in der allein ich gedeihen kann. Der Gegensatz von Egoismus und Altruismus ist damit überwunden: Ganz bin ich nicht als isoliertes Selbst, sondern in der gedeihlichen Wirklichkeit, deren Teil ich bin.“ (ŽıžEK 2005: 38). Darauf, dass Büchner dennoch kritische Distanz zur Beweisführung Spinozas hält, verweist bereits Viëtor, der nachweist, dass die in Büchners Nachlass erhaltenen Handschriften neben Übersetzungen fast ,ausschließlich Notizen und Materialien, zusammengetragen aus philosophischen Handbüchern, vor allem aus der vielbändigen Geschichte der Philosophie des Kantianers Wilhelm Gottliebe Tenemann“ und „kritische Glossen [...] zu Spinozas Ethik“ enthielten (VIËTOR 1949: 247).
} 


\section{Mongelli, B. P. - Zur Inversion des Subjektbegriffs}

dort aufzuzeigen, wo die funktionalistische Methode Eigenheiten behauptet. Eigenheiten, welche nur bei Fischen, aber nicht bei anderen Wirbeltieren vorkommen, bedrohen die Einheitlichkeit, das Übergeordnete, das Urgesetz. So punktuell der Ansatz auch sein mag, so exemplarisch setzt Büchner seine Experimente argumentativ ein. Es ist ein besonderer Verdienst der neuen Marburger Ausgabe, das systematische Vorgehen Büchners in fünf „Kampfzonen' nachzuzeichnen, welche ich hier - ohne die genaue Argumentation, welche den Rahmen dieser Ausführungen sprengen würde - nochmals kurz skizziere: Erstens beweist er gegen Cuvier, den Vater des französischen Funktionalismus, dass die drei Trigeminusäste nicht als ganze, sondern höchstens in einem Nerv, eine Eigenheit der Fische darstellen würden (NS: 263f.). Zweitens sei dieser eine Nerv, der so genannte vierte Ast, „der allein hinreichen würde, um jede vernunftmässige Theorie des Nervensystems unmöglich zu machen“, 7 wohl nur aufgrund unsachgemässen Sezierens als Einheit klassifiziert worden. Dennoch sieht sich Büchner nicht genötigt, alle Abweichungen vom einheitlichen Bauplan, alle Eigenheiten, durch integrative Erklärungen zu beseitigen (NS: 264-267). So kann er drittens diesen vierten Ast, den so genannten Recurrens, zur „genetischen“ Variante erklären (NS: 267-268). Viertens entkräftet er das Argument der Funktionalisten, dass ein besonderer Ast des Trigeminus nur die Fische für die Kiemen hätten. Vielmehr sei dieser Ast nur als „,nerf dérivé“, als „abgeleiteter Nerv“ vom Ohr zu verstehen (NS: 268-269). Und fünftens sei der so genannte Hypoglossus, der unter den Wirbeltieren bisher allein bei den Fischen festgestellt worden sei, auch bei „mehreren Säugethieren“ vorhanden. Zudem beweise gerade dieser Nerv die strukturelle Identität von Rückenmark- und Schädelnerven, da er sich just an der Übergangsstelle beider Systeme befinde (NS: 269-270).

Während in funktionaler Hinsicht primär die Differenz zwischen sensiblen und motorischen Qualitäten der Nerven interessiert, geht es Büchner in seiner genetischen Methode vielmehr um die Einheitlichkeit, die Grundausstattung des Nervensystems bei allen Wirbeltieren. Im Bezug auf den Menschen formuliert er seine Fragen wie folgt in der Probevorlesung:

[W]elche Schädelnerven treten bey den niedrigsten Wirbelthieren zuerst auf, wie verhalten sie sich zu den Hirnmassen und den Schädelwirbeln und nach

\footnotetext{
${ }^{7}$ In der Mémoire heisst es noch: ,[B]ranche qui à elle seule suffirait pour rendre impossible chaque théorie rationnelle du système nerveux" (NS: 38 ).
} 


\section{Mongelli, B. P. - Zur Inversion des Subjektbegriffs}

welchen Gesetzen wird, die Reihe der Wirbelthiere bis zum Menschen, ihre Zahl vermehrt oder vermindert, ihr Verlauf einfacher oder verwickelter? (NS: 161)

Schon aus dieser Versuchsanordnung wird klar, dass zwar eine gewisse Einheitlichkeit des Bauplanes vorausgesetzt wird, dass aber ganz im Sinne der genetischen Methode (,die Reihe der Wirbelthiere bis zum Menschen“ aufsteigend) Varianten möglich sind. ${ }^{8}$ Die Beweisführung steht also ganz unter dem Vorzeichen seiner wissenschaftlichen Exemplarität. Das Beispiel, d.h. das Experimentierobjekt, leistet nicht einfach einen neuen monographischen Beitrag für das riesige Tableau im Sinne der klassischen Repräsentation Foucaults, sondern steht vielmehr für das Ganze. Das empiristisch Feststellbare ist Beweisstück der natura naturans oder, nach Spinoza formuliert, steht das Einzelne immer schon sub specie aeternitatis. Je genauer die Versuchsanlage aufs Einzelne angelegt ist, um es ins Grosse einzugliedern, desto erfolgreicher scheint Büchner zu argumentieren. Man könnte auch sagen, dass sein wissenschaftliches Experiment immer in extremis von Allgemeincharakter einerseits und Spezifizierung andererseits stattfindet, um alles andere dazwischen mitzudenken.

Was bedeutet diese wissenschaftliche Versuchsanordnung nun für den literarischen Büchner, insbesondere für den Woyzeck, an dem er zur selben Zeit arbeitet? Könnte man nicht postulieren, dass er gerade in der Fallgeschichte seines literarischen Experiments - falls man Woyzeck schon so nennen darf - das Aristotelische Ideal gelingender Mimesis, welche allgemein gültigen Charakter hat, gewissermaßen durch die Hintertüre einfängt? Und dies ganz nach derselben Formel: Je spezifischer der casus - hier wohl als Gegengutachten zum Clarus-Gutachten - aufgerollt wird, desto genereller ihre Aussage.

Doch gerade an dieser Stelle werden auch Zweifel laut, kann man doch hinter dieser allgemeinen Vorgehensweise des Experiments eine Abgezwecktheit zweiter Ordnung vermuten. Das Versuchsobjekt begründet seine Teleologie nicht mehr in sich selbst, sondern in der ihm übergeordneten Ordnung. Die Systemimmanenz von Spinozas Ethik wirft ein neues Schlaglicht auf die zentralen Begriffe von Subjekt und Objekt. Das Subjekt des

\footnotetext{
${ }^{8}$ Nicolas Pethes führt diesen Willen für die genetische Methode, den Büchner hier an den Tag legt, auf den deutschen Traditionszusammenhang des Goetheschen Homologiegedankens zurück: „Büchners Behauptung, dass die Schädelnerven aus den Wirbeln des Rückenmarks entstanden seien, beruht aus epistemologischer Sicht nicht allein auf wissenschaftlicher Analyse, sondern auf dem Homologiegedanken, der u.a. auch Goethes Überzeugung eines Kontinuums zwischen Pflanzen, Tier und Mensch zugrunde liegt“ (PETHES 2004: 360).
} 
Mongelli, B. P. - Zur Inversion des Subjektbegriffs

Naturwissenschaftlers, welcher ein Experiment leitet, unterwirft sich seinem System wieder. So wie sein Versuchsobjekt verkommt er selbst zum Objekt einer übergeordneten Ordnung. Müsste man in diesem Moment nicht die Formel nach der „Literatur als Korrektiv“9 bemüßigen, welches diese Ordnung wieder auf den Kopf stellt? Hat das literarische Experiment - wie schon Lenz - einfach ,,auf dem Kopf [zu] gehn“? Oder übernimmt die Literatur sogar die Rolle des Goetheschen „eigne[n] strengste[n] Beobachter[s]“? ${ }^{10}$ Büchner scheint sich zu fragen, was das denn sei, was sich selber wieder zum Objekt macht, um Teil des eigenen Experiments zu werden - wie schon in karikierter Form der Doktor, der in die Sonne schaut, um sein eigenes „Niesen zu beobachten“.

Derselbe Doktor macht zuvor Woyzeck den Vorwurf, seinen Urin nicht zurückgehalten zu haben, worauf dieser einwendet:

WOYZECK: Aber Herr Doktor, wenn einem die Natur kommt.

DOKTOR: Die Natur kommt, die Natur kommt! Die Natur! Hab ich nicht nachgewiesen, dass der musculus constrictor vesicae dem Willen unterworfen ist, WOYZECK der Mensch ist frei, in dem Menschen erklärt sich die Individualität zur Freiheit. Den Harn nicht halten können!

(BÜCHNER 1988: H 4,8, 225)

Diese Erklärung reduziert die Willensfreiheit auf ihre niedrigste Stufe. In ihrer ganzen sozialen Kritik macht sie auch deutlich, wie ein politisches Schlagwort der Aufklärung auf tiefste Ebene zurückgestuft werden kann. Wenn der freie Willen nur da ist, um die Grundbedürfnisse zu unterdrücken, so nützt er gar nichts. Im Gegenteil: Er wird nur zum Mittel des Herrschenden, damit wieder in eine Teleologie eingebunden und dadurch obsolet. Die Kombination der Parodie auf den freien Willen mit der Niesepisode - welche wohlweislich die angedeutete Grundlagenforschung aus dem Manuskript H2 ersetzt verdeutlicht aber noch etwas Anderes: nämlich die Doppelbödigkeit des Naturbegriffs einerseits als Ebene des Affekts und des instinktiven neuronalen Reflexes, andererseits als Ausgangs- und Kulminationspunkt einer philosophischen Richtung von vergleichender Anatomie. So figuriert in den wissenschaftlichen Arbeiten, vorab in der mémoire, die Barbe

\footnotetext{
${ }^{9}$ So der Titel des einleitenden Aufsatzes von Karl RICHTER zum zweiten Teil des Sammelbandes, welcher die Zeitperiode von 1830 bis 1890 umfasst. (RICHTER 1997: 131-138).

${ }^{10}$ GOETHE 1982: 6.
} 
Mongelli, B. P. - Zur Inversion des Subjektbegriffs

als ,primitiver Typus“"11, worauf sich die ,genetisch“ angelegte Analogieforschung quer durch alle Wirbeltierklassen bezieht. Mit anderen Worten: die konkreten, fallspezifischen Parodien im literarischen Experiment, welche in der Sekundärliterliteratur ständig bemüßigt werden, um biographische Parallelen zu bestimmten Dozenten, welche Büchner in Gießen erlebte, herzustellen, ${ }^{12}$ diese Parodien sind letztlich nur amüsante Manövriermasse, hinter der sich der Grundtypus Woyzeck versteckt hält. Er ist derjenige, der „philosophiert“; er ist wohl der einzige Mensch im ganzen Drama, obwohl es mit ihm nur bergab geht. ${ }^{13}$ Denn er verweist auf die intrinsisch angelegte Spur der Natur, welche gelesen werden will: „Haben sie [sic] schon gesehn in was für Figurn die Schwämme auf d. Boden wachsen. Wer das lesen könnt.“14 Gleichzeitig verweist Büchner auch in der Einleitung seiner Probevorlesung auf die Lesbarkeit der Natur nach ästhetischem Prinzip, wenn er sagt:

Hat man auch nichts Ganzes erreicht, so kamen doch zusammenhängende Strecken zum Vorschein und das Auge, das an einer Unzahl von Thatsachen ermüdet, ruht mit Wohlgefallen auf so schönen Stellen, wie die Metamorphose der Pflanze aus dem Blatt, die Ableitung des Skeletts aus der Wirbelform; die Metamorphose ja die Metempsyche des Fötus während des Fruchtlebens; die Repräsentationsidee Oken's in der Klassifikation des Thierreichs u. d. gl. m. In der vergleichenden Anatomie strebte Alles nach einer gewissen Einheit, nach dem Zurückführen aller Formen auf den einfachsten primitiven Typus. (NS, 155,32-47)

Nicht nur im Woyzeck, sondern auch in der Wissenschaft wird die Natur zum Text, in dem man auf schöne Stellen aufmerksam wird, welche den eigentlichen Zusammenhang herstellen. ${ }^{15}$ Erst seine „aberratio mentalis partialis“ erlaubt es dem Hauptprotagonisten des Dramas, aus der ganzen Versuchsanlage des Doktors, welche - salopp formuliert ökonomische Einsparungen (,Hülsenfrüchte statt Fleisch für die Soldaten') bezweckt, ${ }^{16}$ und

\footnotetext{
11 „Je ne veux que démontrer le type primitif, d'après lequel ces parties se sont développées“ (NS 100).

12 Vgl. dazu VIËTOR 1949: 218, KUBIK 1991 und FRICK 2004: 266.

13 „Hier geht es nicht um die Besonderheit eines Falles [...], siehe da, ein Mensch!“( (VIËTOR 1949: 218).

${ }^{14}$ BÜCHNER 1988: H4,8, 225.

${ }^{15}$ Vgl. dazu MÜLler NiElaba 2000.

16 In diesem Fall handelt es sich nicht um eine Versuchsanlage, welche Büchner von Justus von Liebig abgeschrieben hat, der zu Büchners Studienzeit in Gießen ,die gleichen ernährungsphysiologischen Experimente an Soldaten der großherzoglichen Leibkompagnie durchgeführt" habe - so Buddecke (in BORNSCHEUER 1972: 15), sondern vielmehr um ein Konglomerat der anfangs der 1830er Jahren relevanten ernährungsphysiologischen Fragen zusammen: „Das Drama präsentiert naturwissenschaftliche wie medizinische, anatomische wie
} 
Mongelli, B. P. - Zur Inversion des Subjektbegriffs

aus den Schicksalsschlägen des Lebens (Woyzeck als Eifersuchtsdrama) wenigstens temporal partiell auszubrechen. So wird er nicht nur für den Doktor zum ,interessante[n] casus“ sondern auch ganz allgemein, systemtheoretisch gesprochen, auf zweiter Ebene. Dies unterstreicht die Apposition „Subjekt Woyzeck“: trotz vielleicht sarkastischen Beigeschmacks bildet die Hauptfigur in ihrer doppeldeutigen Rolle eine Scharnierfunktion zwischen Versuchsobjekt (im Sinne des sub-jectum, des dem Experiment Unterworfenen) und autonomem Individuum, welches - in seiner ganzen Geworfenheit in diese Welt - diese trotzdem in ihrer doppelten Natur wahrnimmt. Erst in dieser Doppeldeutigkeit, in dieser Unentschiedenheit „wege des Gedankestrichels zwische Ja, und nein ja - und nein“ (H2, 7, 216) tut sich im System - gerade auch in seiner Abgezwecktheit zweiten Grades, systemtheoretisch gesprochen - etwas auf, das von einem freien Willen zeugen könnte.

Aus diesem Grund drängen sich literarischer und wissenschaftlicher Diskurs an den Rand ihrer Möglichkeiten. So schafft die experimentelle (und teleologische) Anlage der Probevorlesung, die darin fast vollumfänglich der Dissertation entspricht, die lebensnotwendige Verbindung, auf die Büchner anspielt, wenn er in der Einleitung die prekäre Situation der Philosophie a priori skizziert. Was am neu formulierten Anfang der Probevorlesung noch als schiere Unmöglichkeit dargestellt wird, ermöglicht die experimentelle Beweisführung, welche zuvor in der Dissertation auf die Autorität der rhetorischen Setzung in der partie philosophique angewiesen war. Diese Umkehrung von Allgemeinbetrachtung und Beweisführung hat zur Folge, dass ein vorgegebener Weg nur noch nachgezeichnet werden muss. Parallel dazu bewegt sich Woyzeck in seinem sozialen Feld: Je mehr er an dessen Rand gedrängt wird, je verzweifelter seine Lage ist, desto mehr wird er zum Menschen. Die teleologische Konsequenz, welche dem experimentellen Teil der Probevorlesung innewohnt und den Menschen innerhalb des ganzen Natursystems philosophisch begründet, ist zwar von fatalistischer Konsequenz, fördert aber gleichzeitig die Freiheit innerhalb ihrer Begründung wieder zu Tage. Man kann es drehen und wenden, wie man will: Sowohl die Konsequenz der wissenschaftlichen Begründung wie auch die unabänderliche schiefe Bahn, auf welche Woyzeck gerät, erfordern Darstellungsmittel, welche in ihrer Diskontinuität über die fatalistische Einbahnstrasse hinausweisen - seien es

physiologische Sachverhalte, an Beispielen konkretisiert und terminologisch wie historisch korrekt. Woyzeck ist somit auch ein medizinhistorisches Dokument"“ (ROTH 2000: 519). 
Mongelli, B. P. - Zur Inversion des Subjektbegriffs

die in sich oft unabgeschlossenen Szenenfragmente im Woyzeck oder die spezifischen Beispiele der Dissertation. Je mehr diese ihr Eigenleben beweisen, desto weniger treten sie den Beweis von fatalistischer Konsequenz an, zumal die „unmittelbare Anwendung [...] zum Beweis irgendeiner Hypothese schädlich ist" (GoETHE 1982: 6). Erst die Zweckfreiheit, ja die Objektivierung des Experiments, das im Woyzeck neben dem (abgesehen vom Auftritt des Professors) gar nicht so sarkastischen, jedenfalls gut begründeten naturwissenschaftlichen Diskurs andere Stimmen zulässt oder die „philosophische Manifestation eines Urgesetzes“ wie in der Probevorlesung miteinbezieht, erlaubt den entsprechenden gesellschaftspolitischen Befreiungsschlag. Oder anders formuliert: Erst die politische Sensibilisierung im Vormärz für die Rhetorizität von Wissenschaft und Literatur erlaubt eine kritische Reflexion der Abhängigkeit und Autonomie des schillernden „Subjects der Geschichte“.

\section{Literaturverzeichnis}

BORNSCHEUER, Lothar (Hg.). Erläuterungen und Dokumente. Woyzeck. Stuttgart, Reclam, 1972

BüCHNER, Georg. Werke und Briefe. Herausgegeben von Karl Pörnbacher, Gerhard Schaub, Hans-Joachim Simm und Edda Ziegler. München, Carl Hanser, 1988.

BüCHNER, Georg. Naturwissenschaftliche Schriften. Marburger Ausgabe, Marburg 2008 (= NS).

FRICK, Werner. „Und sehe, dass wir nichts wissen können...“. Poetische Wissenschaftsskepsis bei Goethe, Kleist und Büchner. In: Norbert Elsner und Werner Frick (Hgg.): „Scientia poetica“. Literatur und Wissenschaft. Göttingen, Wallstein 2004, 243-272.

GoETHE, Johann Wolfgang. Der Versuch als Vermittler. In: Schriften zur Naturwissenschaft. Stuttgart, Reclam, 1982, 6.

KUBIK, Sabine. Krankheit und Medizin im literarischen Werk Georg Büchners. Stuttgart, M\&P, 1991.

LuHMann, Niklas: Die Wissenschaft der Gesellschaft. Frankfurt am Main, Suhrkamp, 1990.

Müller NielabA, Daniel. „Das Auge ruht mit Wohlgefallen auf so schönen Stellen.“ Büchners Nerven-Lektüre. In: Weimarer Beiträge 46. Weimar, 2000, 325-345.

Müller-Sievers, Helmut. Desorientierung. Anatomie und Dichtung bei Georg Büchner. Göttingen, Wallstein, 2003.

Pethes, Nicolas. Poetik/Wissen. Konzeptionen eines problematischen Transfers. In: Burghard Dedner und Thomas Michael Mayer (Hg.): Romantische Wissenspoetik. Die Künste und die Wissenschaften um 1800. Würzburg, Königshausen und Neumann, 2004, 341-372. 
Mongelli, B. P. - Zur Inversion des Subjektbegriffs

Rотн, Udo. Georg Büchners Woyzeck als medizinhistorisches Dokument. In: Georg Büchner Jahrbuch 9. Tübingen, Niemeyer, 2000, 503-519.

Richter, Karl / SchÖnert, Jörg / TitzMAnn, Michael (Hg.). Die Literatur und die Wissenschaften 1770-1930. Stuttgart, Metzler, 1997, 131-138.

VIËTOR, Karl. Georg Büchner. Politik. Literatur. Wissenschaft. Bern, Francke, 1949.

ŽIŽEK, Slavoj. Die politische Suspension des Ethischen. Frankfurt am Main, Suhrkamp, 2005. 